\begin{tabular}{|c|c|}
\hline Title & Effect of warm-up exercise on delay ed-onset muscle soreness \\
\hline Author(s) & Takizawa, Kazuki; Soma, Toshio; Nosaka, Kazunori; Ishikawa, Tomoji; Ishii, Kojiro \\
\hline Citation & $\begin{array}{l}\text { European Journal of Sport Science, 12(6), } 455-461 \\
\text { https://doi.org/10.1080/17461391.2011.566371 }\end{array}$ \\
\hline Issue Date & $2011-08-22$ \\
\hline DOC URL & http:/hdl.handle.net/2115/50707 \\
\hline Rights & $\begin{array}{l}\text { This is an A uthor's A ccepted Manuscript of an article published in European Journal of Sport Science, 12(6), pp.455- } \\
461,22 \text { Aug. 2011, copyright Taylor \& Francis, available online at: } \\
\text { http:/www.tandfonline.com } 10.1080 / 17461391.2011 .566371 .\end{array}$ \\
\hline Type & article (author version) \\
\hline File Information & EJSS12-6_455-461.pdf \\
\hline
\end{tabular}

Instructions for use 
TEJS-2010-0389-R1

\section{EFFECT OF WARM-UP EXERCISE ON DELAYED-ONSET MUSCLE SORENESS}

Running head: Warm-up and DOMS

Kazuki TAKIZAWA ${ }^{1)}$, Toshio SOMA ${ }^{2)}$, Kazunori NOSAKA ${ }^{3)}$, Tomoji ISHIKAWA ${ }^{4)}$, Kojiro ISHII ${ }^{5)}$

${ }^{1)}$ Institute for the Advancement of Higher Education, Hokkaido University, Japan

${ }^{2)}$ Department of Physical Therapy, Niigata University of Health and Welfare, Japan

3) School of Exercise, Biomedical and Health Sciences, Edith Cowan University, Australia

4) Department of Health and Sports, Niigata University of Health and Welfare, Japan

${ }^{5)}$ Faculty of Health and Sports Science, Doshisha University, Japan

Corresponding author:

Kazuki Takizawa

Institute for the Advancement of Higher Education, Hokkaido University

Hokkaido University Sports Training Center,

Kita 17 Nishi 13, Kita-ku, Sapporo, Hokkaido, Japan

Tel \& Fax: +81-11-706-5591

E-mail: takizawa@high.hokudai.ac.jp 


\begin{abstract}
This study investigated whether a warm-up exercise consisting of 100 submaximal concentric contractions would attenuate delayed-onset muscle soreness (DOMS) and decreases in muscle strength associated with eccentric exercise-induced muscle damage. Ten male students performed two bouts of the elbow flexor exercise consisting of 12 maximal eccentric contractions with a warm-up exercise for one arm (WU) and without warm-up for the other arm (control: $\mathrm{CON}$ ) in a randomised, counterbalanced order separated by 4 weeks. Muscle temperature of the biceps brachii prior to the exercise was compared between the arms, and muscle activity of the biceps brachii during the exercise was assessed by surface integral electromyogram (iEMG). Changes in visual analog scale for muscle soreness and maximal voluntary isometric contraction strength (MVC) of the elbow flexors were assessed before, immediately after, and every 24 hours for 5 days following exercise, and compared between the WU and CON conditions by a two-way repeated measures ANOVA. The pre-exercise biceps brachii muscle temperature was significantly $(\mathrm{p}<0.01)$ higher for the WU $\left(35.8 \pm 0.2^{\circ} \mathrm{C}\right)$ than $\mathrm{CON}$ condition $\left(34.4 \pm 0.2^{\circ} \mathrm{C}\right)$, but no significant differences in iEMG and torque produced during exercise were evident between conditions. Changes in muscle soreness and MVC were not significantly different between conditions, although these variables showed significant $(\mathrm{p}<0.05)$ changes over time. It was concluded that the warm-up exercise was not effective in mitigating DOMS and loss of muscle strength following maximal eccentric exercise.
\end{abstract}

Key words: muscle damage, eccentric exercise, muscle temperature, electromyogram (EMG), deep body thermometer 


\section{INTRODUCTION}

Muscle pain develops after unaccustomed exercise and persists for several days, which is referred to as the delayed-onset muscle soreness (DOMS). Although its aetiology has not been fully understood, it has been documented that mechanical damage and subsequent inflammatory responses to muscle fibres and/or connective tissue are associated with DOMS (Clarkson and Hubal, 2002; Cheung et al., 2003, Connolly et al., 2003; Nosaka et al., 2003). To prevent or attenuate DOMS, many interventions including cryotherapy, ultrasound, electric stimulation, massage, stretching, and immobilization have been examined; however, none of these modalities have been shown to be strongly effective (Cheung et al., 2003; Connolly et al., 2003).

Warm-up exercise is generally performed before exercise in the belief that it improves exercise performance and prevents or attenuates DOMS; however, controversy exists concerning its efficacy on DOMS. For example, High and Howley (1989) reported no effect of static stretching alone or a combination of static stretching and warm-up exercise (10 min stepping exercise using a 10-cm height box) on muscle soreness induced by an exhaustive stepping exercise using a higher box (40-cm for women, $50-\mathrm{cm}$ for men). On the other hand, Law and Herbert (2007) reported that muscle soreness after a 30 min of backward downhill walking was approximately $20 \%$ less for the group that performed a warm-up exercise (10 min uphill walking) compared with the group that did not perform the warm-up exercise.

Using an eccentric exercise model of the elbow flexors, three studies have shown different findings for the effect of warm-up exercise on DOMS and other markers of muscle damage. Rodenburg et al. (1994) reported that a combination of warm-up exercise (15 minutes arm cranking exercise at 60\% HRmax) and stretching before exercise, and massage after exercise was effective for attenuating DOMS after eccentric exercise; however, the warm-up exercise alone did not provide such effect. Evans et al. (2002) compared five 
groups of subjects consisting of 16 men and 27 women; low-heat passive heating, high-heat passive heating, active warm-up exercise consisting of 100 submaximal concentric contractions of the elbow flexors, eccentric exercise only and control groups, for changes in muscle soreness, isometric strength, arm circumference, plasma creatine kinase (CK) activity and range of motion (ROM) following 50 maximal eccentric contractions. They reported that only the high-heat passive heating group showed attenuation of the changes in arm circumference, ROM and muscle soreness compared with other groups, but did not find any prophylactic effect of the active warm-up exercise on any of the parameters. In contrast, using a cross-over design in which one arm of five young women was assigned to a warm-up exercise condition, and the other arm was for a control condition, Nosaka and Clarkson (1997) showed that 100 submaximal concentric contractions of the elbow flexors reduced the magnitude of DOMS and other symptoms of muscle damage (maximal voluntary isometric contraction strength, range of motion, upper arm circumference and plasma CK activity) after 12 maximal eccentric contractions. They concluded that the concentric exercise provided a warm-up effect to attenuate eccentric exercise induced damage.

It should be noted that the warm-up exercise protocol of the study by Nosaka and Clarkson (1997) was similar to that of the aforementioned study by Evans et al. (2002), but the two studies report conflicting findings. It may be that the differences in the eccentric exercise protocol (12 versus 50 maximal eccentric contractions) and study design (cross-over versus group comparison) were associated with the different findings between the studies; however, no clear explanation can be made. Since the study by Nosaka and Clarkson (1997) reported a strong prophylactic effect of the warm-up exercise on eccentric exercise-induced muscle damage, the study should be replicated by using a larger sample size and different sex. It is also important to include muscle temperature and muscle activity measurements, which were not performed in the previous study (Nosaka and Clarkson, 1997), since the magnitude 
of increase in muscle temperature may affect the warm-up effect on muscle damage markers, and muscle activity assessed by surface electromyogram (EMG) during eccentric contractions may be influenced by warm-up exercise.

Therefore, the purpose of this study was to investigate changes in muscle temperature after a warm-up exercise and to test the hypothesis that a warm-up exercise consisting of repetitive submaximal concentric contractions before eccentric exercise would attenuate the extent of DOMS and decrease in muscle strength following maximal eccentric exercise of the elbow flexors. We hypothesised that warm-up exercise would attenuate DOMS and decreases in muscle strength following exercise.

\section{METHODS}

\section{Subjects}

Ten male college students (mean \pm SD age: $18.8 \pm 0.5 \mathrm{yrs}$, height: $173.6 \pm 6.1 \mathrm{~cm}$, weight: $62.3 \pm 7.9 \mathrm{~kg}$ ) participated in this study after signing a written informed consent form according to the Declaration of Helsinki. All subjects were physically active and participated in recreational sport activities; however, none of them had been involved in a resistance training program for at least 6 months prior to the participation in the present study. All subjects were free from musculoskeletal disorders and did not have any inflammatory diseases. They were instructed not to take anti-inflammatory drugs and any therapeutic interventions during the experimental period and asked maintain their regular diet during the experiment periods. The study was approved by the Institutional Ethics Committee.

\section{Experimental design}

All subjects performed two bouts of eccentric exercise of the elbow flexors with warm-up exercise (WU) for one arm and without warm-up exercise (control: CON) for the 
contralateral arm separated by 4 weeks. The use of dominant and non-dominant arms, and the order of the conditions were randomised and counterbalanced amongst subjects. The biceps brachii temperature was measured immediately before the eccentric exercise for both conditions as described below. Muscle activity of the biceps brachii during the exercise was assessed by surface EMG, and the torque produced in the eccentric exercise was recorded. The dependent variables included muscle soreness using a visual analogue scale (VAS) and maximal voluntary isometric contraction (MVC) strength of the elbow flexors. The present study focused on these two measures, since these are directly associated with the symptoms of muscle damage that influence athletic performance and daily activities. These measures were taken before, immediately after, and every 24 hours for 5 days following exercise, and changes in these variables were compared between the WU and CON conditions.

\section{Warm-up exercise}

Warm-up exercise consisted of 100 concentric contractions of the elbow flexors based on the previous studies (Nosaka and Clarkson, 1997; Evans et al., 2002). An isokinetic dynamometer (System 3, Biodex Medical Systems, U.S.A.) was used for the exercise, and each subject was positioned on the chair of the dynamometer with $45^{\circ}$ shoulder flexion, and the forearm was positioned in front of the body with the wrist secured to the dynamometer's lever arm. The gravity correction of the dynamometer was made at $90^{\circ}$ of elbow joint angle before according to the instructions in the manual. The forearm was moved from a fully extended $\left(180^{\circ}\right)$ to a flexed position $\left(30^{\circ}\right)$ at the angular velocity of $120^{\circ} \cdot \mathrm{s}^{-1}$ with a 10 -s rest between contractions. The subjects were instructed to generate minimal force to move the lever arm for concentric contractions, and to relax and let the arm return to the extended position without force generation after each concentric contraction. During the concentric contractions, the force production was less than $5 \mathrm{Nm}$ for all contractions of all subjects. 
The warm-up exercise was completed 5 min before the eccentric exercise described below.

\section{Eccentric exercise}

Each subject performed eccentric exercise the elbow flexors using the isokinetic dynamometer, and was positioned on the isokinetic equipment in the same way as that of the warm-up exercise. The subject was instructed to maximally resist the movement of the lever arm of the dynamometer in which the subject's elbow joint was forcibly extended from an elbow-flexed $\left(30^{\circ}\right)$ to an elbow-extended $\left(180^{\circ}\right)$ position at the angular velocity of $30^{\circ} \cdot \mathrm{s}^{-1}$. This was repeated 12 times with a 15-s rest between contractions (Nosaka and Clarkson, 1997). The peak torque generated in each eccentric contraction was detected by a software programme of the dynamometer (Biodex Advantage Software version 3.33, Biodex Medical Systems, U.S.A.).

\section{Muscle temperature}

The muscle temperature of the biceps brachii was measured by a deep body thermometer (Core temp CTM-210, Telmo, Japan) before the warm-up exercise for the WU condition and before the eccentric exercise for both conditions. The deep body thermometer is based on the "zero heat flow" technique and its measurement principle has been explained in details by Togwa et al. (1976). The validity of the method has been established (Togwa et al., 1976), and it has been reported that the values obtained by the deep body thermometer are highly $\left(r^{2}=0.87\right)$ correlated with the values measured by a needle thermometer at $18-\mathrm{mm}$ depth (Matsukawa et al., 1996). In the present study, a circular sensor probe of the deep body thermometer (20 $\mathrm{mm}$ in diameter) was placed at the mid-belly of the biceps brachii for at least 5 minutes before each measurement, since the initial response time for the thermometer to reach the deep body temperature takes approximately 5 minutes and quickly 
respond the temperature change thereafter (Yamakage et al., 2002). In the WU condition, the sensor probe was kept on the muscle during the warm-up exercise. The pre-exercise muscle temperature was determined 1 min before the eccentric exercise for both conditions, which was 4 min after the warm-up exercise for the WU condition.

\section{Electromyogram (EMG)}

Muscle activity of the biceps brachii during the eccentric exercise was assessed by surface EMG. After rubbing the skin with an alcohol swab to reduce the surface impedance less than $5 \mathrm{k} \Omega$, two $\mathrm{Ag} / \mathrm{AgCl}$ electrodes (diameter: $10 \mathrm{~mm}$ ) were placed $20 \mathrm{~mm}$ apart on the midline of the biceps brachii; half way between the centre of the belly and myotendinous junction, and a ground electrode was placed at the elbow joint. The EMG signals were amplified by a pre-amp (DPA-10P, Dia Medical System Co., Ltd., Japan) and an amplifier (DPA-2016, Dia Medical System Co., Ltd., Japan), and recorded at 2,000Hz to a computer (Endeavor, Epson, Japan) through an A/D converter (Power Lab 16/s, AD Instruments, Australia) with a $10 \mathrm{~Hz}$ high-pass filter. The integral EMG (iEMG) was calculated by a software (Chart, AD Instruments, Australia), and expressed as a percentage to the iEMG during the pre-exercise maximal isometric contraction of the elbow flexors at the elbow angle of $90^{\circ}$.

\section{Visual analogue scale (VAS)}

Muscle soreness of the biceps brachii was assessed by a VAS of a 100-mm line with "no pain" at one end and "extremely sore" at the other while applying pressure to the muscle. The same investigator took all muscle soreness assessments, and one finger was placed on the middle of the biceps brachii and pressure was applied toward the deeper tissues for approximately $3 \mathrm{~s}$. 


\section{Maximal voluntary isometric contraction torque (MVC)}

Each subject was positioned in the same way to that of the eccentric exercise as explained above. The subjects performed two maximal voluntary isometric contractions for 5-s with a 60-s rest between contractions on the isokinetic dynamometer, and the peak torque was obtained from each contraction, and the higher value was used for further analysis. The test-retest reliability of this measure was established prior to the experiment using two pre-exercise measures at two different days, and the coefficient of variation of the measure was $3.6 \%$, and Intraclass correlation $(\mathrm{R})$ was 0.98 .

\section{Statistical analysis}

The pre-exercise muscle temperature was compared between the WU and CON conditions by a paired Student's t-test. Changes in \%iEMG and torque over 12 eccentric contractions were compared between conditions by a two-way repeated measured ANOVA. Changes in muscle soreness (VAS) and MVC over time were also compared between conditions by a two-way repeated measured ANOVA. When a significant time effect was found, a Student's t-test with Bonferroni correction was followed to compare between the pre-exercise value and the post-exercise values. Comparisons between the first and second eccentric exercise bouts regardless of the conditions were also made for changes in \%EMG and torque over 12 eccentric contractions and changes in muscle soreness and MVC over time by a two-way repeated measures ANOVA. A significance level was set at $p \leq 0.05$ for the ANOVAs, and at $0.05 / 6=0.008$ for the post-hoc test, because of the multiple comparisons of 6 time points. All results are shown as the mean \pm SD. 


\section{RESULTS}

\section{Muscle temperature}

The biceps brachii muscle temperature was $34.8 \pm 0.1{ }^{\circ} \mathrm{C}$ before warm-up exercise for the WU condition, and significantly $(\mathrm{P}<0.01)$ increased to $35.8 \pm 0.2{ }^{\circ} \mathrm{C}$ after the warm-up and immediately before the eccentric exercise. The pre-exercise muscle temperature was significantly $(\mathrm{P}<0.01)$ higher for the WU compared with the $\mathrm{CON}$ condition $\left(34.4 \pm 0.2^{\circ} \mathrm{C}\right)$.

\section{\%iEMG and torque}

Figure 1 shows changes in \%iEMG and peak torque over 12 eccentric contractions. The \%iEMG did not change significantly over time, and no significant difference between the WU and CON conditions was evident for the changes and the average values. Changes in the torque generated during eccentric contractions were not significantly different between conditions, and no significant condition effect was found for the average torque values between conditions. When comparing the first and second bouts regardless of the conditions, no significant difference in the changes in MVC over 12 eccentric contractions was evident.

\section{Muscle soreness}

Muscle soreness developed after eccentric exercise, and peaked 2-3 days post-exercise (Figure 2). No significant differences in the changes were evident between the WU and CON conditions (Figure 2). And, no significant difference between bouts was found for the changes in muscle soreness.

\section{MVC}

Pre-exercise MVC was not significantly different between the WU $(42.4 \pm 8.3 \mathrm{Nm})$ and $\mathrm{CON}(39.2 \pm 11.1 \mathrm{Nm})$ conditions. No significant difference in the changes in MVC was 
evident between conditions (Figure 3). MVC (average value for both conditions) decreased by $25.8 \pm 3.4 \%$ immediately after exercise, and was still significantly lower than the baseline by $18.4 \pm 4.2 \%$ at 5 days post-exercise (Figure 3). Changes in MVC following exercise were not significantly different between the first and second bouts.

\section{DISCUSSION}

The results of this study showed that the warm-up exercise increased muscle temperature by $1.4^{\circ} \mathrm{C}$, but did not affect the torque and iEMG during eccentric exercise, and the changes in muscle soreness and MVC following the exercise. Thus, the findings of the previous study by Nosaka and Clarkson (1997) reporting that 100 submaximal concentric contractions of the elbow flexors performed prior to maximal eccentric exercise of the elbow flexors attenuated DOMS and loss of muscle strength were not reproduced in the present study.

The present study used the same warm-up exercise protocol (100 submaximal concentric contractions) as that of the previous study by Nosaka and Clarkson (1997), and the eccentric exercise protocol (12 maximal eccentric contractions) was similar between the studies. A slight difference in the eccentric exercise was that the previous study used an arm curl machine and the subjects were asked to resist against the force generated by the investigator, whereas the present study used an isokinetic dynamometer. However, the magnitude of decrease in maximal isometric strength immediately after exercise was comparable (approximately $25 \%$ ) between the studies. Thus it seems unlikely that the difference in the exercise protocol was the main reason for the different findings between the studies.

In the study by Nosaka and Clarkson (1997), muscle temperature measures were not performed, so the changes in the biceps brachii muscle temperature after the warm-up 
exercise were unknown. In the present study, the biceps brachii muscle temperature increased by $1.4^{\circ} \mathrm{C}$ after the warm-up exercise. Probably, the increase in the muscle temperature in the study by Nosaka and Clarkson (1997) was similar to this, since the warm-up exercise protocol was similar. Evans et al. (2002) reported that an active warm-up exercise consisting of 100 submaximal concentric contractions of the elbow flexors did not attenuate DOMS following 50 maximal eccentric contractions of the elbow flexors, but a high-heat passive heating mitigated muscle soreness. They showed that the increase in muscle temperature after low-heat passive heating, high-heat passive heating, and active warm-up exercise was $1^{\circ} \mathrm{C}, 3.5^{\circ} \mathrm{C}$, and $1^{\circ} \mathrm{C}$, respectively. It should be noted that they examined the changes in biceps brachii muscle temperature (2 $\mathrm{cm}$ depth) after each intervention in a separate study. They stated that the elevated muscle temperature by $1^{\circ} \mathrm{C}$ in the warm-up exercise might not be enough to produce a significant protective effect as shown in the high-heat passive heating. When looking at the other previous studies, High and Howley (1989) reported that warm-up exercise (stepping exercise at 2.7 METs) before a step exercise to exhaustion did not reduce DOMS, but Law and Herbert (2007) reported that warm-up exercise (uphill walking at 3.1- 3.4 METs) before a 30-min backward downhill walking reduced DOMS by $20 \%$. These studies did not measure muscle temperature, thus it is not known whether the different findings of the studies can be explained by a difference in pre-exercise muscle temperature. It is possible that the extent of increase in muscle temperature by the warm-up exercise in the present study may be not high enough to produce a reduction in DOMS.

The results of the present study also showed that the warm-up exercise did not affect the changes in MVC (Figure 3). Nosaka and Clarkson (1997) reported that muscle strength fully recovered to the baseline by 5 days post exercise for the warm-up condition, but was more than $10 \%$ lower than the baseline for the control condition at the same time point. In 
the present study, MVC was still significantly lower than the baseline by $18 \%$ at 5 days post-exercise for both conditions, which was greater than the decrease reported in the study by Nosaka and Clarkson (1997) in spite of the similar decrease in MVC immediately after exercise. Thus, it is possible that the magnitude of muscle damage, judging from the prolonged decreases in MVC, was greater in the present study than that of the previous study. In the study by Evans et al. (2002), a larger number of eccentric contractions of the elbow flexors (i.e. 50 contractions) were performed than that of the present study (i.e., 12 contractions), and they did not find any effect of the warm-up exercise on decrease and recovery of muscle strength. It may be that the prophylactic effect of warm-up exercise on muscle damage is dependent on the magnitude of muscle damage induced by eccentric exercise.

It should be noted that Nosaka and Clarkson (1997) used 5 female subjects, but the present study used 10 male subjects. A previous study has reported that men are more susceptible to eccentric exercise-induced muscle damage than women (Kerksick et al., 2008). It might be that a sex difference exists for the effect of warm-up exercise on eccentric exercise-induced damage. It is important to note that the torque generated during exercise in this study was not significantly different between the WU and CON conditions, and no significant difference in iEMG was evident between the conditions (Figure 1). Thus, it does not appear that the warm-up exercise used in the present study improved exercise performance. These would indicate that mechanical stress to the muscle fibres during the exercise was similar between the conditions. It has been documented that the intensity of eccentric contractions is a predictor for the magnitude of muscle damage (Nosaka et al., 2003). Therefore, it seems possible that warm-up exercise has little effect on symptoms of eccentric exercise-induced muscle damage, when the intensity eccentric exercise is high.

It is generally believed that warming-up muscles is beneficial for preventing 
exercise-induced injuries (Woods et al., 2007). However, this does not appear to be the case for eccentric exercise-induced muscle damage. Previous studies (High and Howley, 1989; Evans et al., 2002) also failed to find positive effects of a warm-up exercise on eccentric exercise-induced muscle damage. Thus, it does not appear that a warm-up exercise consisting of concentric contractions resulting in a small increase in muscle temperature can produce a prophylactic effect. A different type of warm-up exercise that includes stretching would attenuate DOMS and muscle damage as shown in a previous study (Law and Herbert, 2007; Rodenburg et al., 1994). Even if warm-up exercise is not effective for prevention of DOMS and eccentric exercise-induced muscle damage, this does not necessarily deny the importance of warm-up exercise, since warm-up exercise is shown to be effective for preventing injuries (Woods et al., 2007) and improving exercise performance (Bishop, 2003).

In summary, the present study showed that DOMS and decrease in muscle strength following 12 maximal eccentric contractions of the elbow flexors were not different between conditions with and without warm-up exercise consisting of 100 repetitive submaximal concentric contractions that increased biceps brachii muscle temperature by $1.4^{\circ} \mathrm{C}$. It is concluded that the warm-up exercise consisting of submaximal concentric contractions has little effect on DOMS and decrease in muscle strength following eccentric exercise. Further studies are necessary to investigate other types of warm-up exercise such as combination of concentric contractions and stretching for their effect on DOMS and eccentric exercise-induced muscle damage.

\section{Acknowledgment}

The authors are grateful to the subjects for their participation. This work was supported by Japan Society for the Promotion of Science (KAKENHI), Grant-in-Aid for Young Scientists (B), No. 19700511. 


\section{REFERENCES}

Bishop, D. (2003) Warm up I. Potential mechanisms and the effects of passive warm up on exercise performance. Sports Medicine, 33, 439-454.

Cheung, K., Hume, P. A., Maxwell, L. (2003) Delayed onset muscle soreness: treatment strategies and performance factors. Sports Medicine, 33, 145-164.

Clarkson, P. M., Hubal, M. J. (2002) Exercise-induced muscle damage in humans. American Journal of Physical Medicine and Rehabilitation, 81, S52-S69.

Connolly, D. A. J., Sayers, S. P., McHugh, M. P. (2003) Treatment and prevention of delayed onset muscle soreness. Journal of Strength and Conditioning Research, 17, 197-208.

Evans, R. K., Knight, K. L., Draper, D. O., Parcell, A. C. (2002) Effects of warm-up before eccentric exercise on indirect markers of muscle damage. Medicine and Science in Sports Exercise, 34, 1892-1899.

High, D. M., Howley, E. T. (1989) The effects of static stretching and warm-up on preventions of delayed-onset muscle soreness. Research Quarterly for Exercise and Sports, 60, $357-361$.

Kerksick, C., Taylor, L. 4th, Harvey, A., Willoughby, D. (2008) Gender-related differences in muscle injury, oxidative stress, and apoptosis. Medicine Science in Sports Exercise, 40, 1772-1780.

Law, R. Y. W., Herbert, R. D. (2007) Warm-up reduces delayed-onset muscle soreness but cool-down does not: a randomised controlled trial. Australian Journal of Physiotherapy, 53, 91-95.

Matsukawa, T., Kashimoto, S., Ozaki, M. Shindo, S., Kumazawa, T. (1996) Temperatures measured by a deep body thermometer (Coretemp) compared with tissue temperatures measured at various depth using needle placed into the sole of the foot. European Journal of Anesthesiology, 13, 340-345. 
Nosaka, K., Clarkson, P. M. (1997) Influence of previous concentric exercise on eccentric exercise-induced muscle damage. Journal of Sports Sciences, 15, 477-483.

Nosaka, K., Lavender, A., Newton, M., Sacco, P. (2003) Muscle damage in resistance training: Is muscle damage necessary for strength gain and muscle hypertrophy? International Journal of Sports and Health Science, 1, 1-8.

Rodenburg, J. B., Steenbeek, D., Schiereck, P., Bar, P. R. (1994) Warm-up, stretching and massage diminish harmful effects of eccentric exercise. International Journal of Sports Medicine, 15, 414-419.

Togwa, T., Nemoto, T., Yamazaki, T., Kobayashi, T. (1976) A modified internal temperature measurement device. Medicine and Biological Engineering, 14, 361-364.

Woods, K., Bishop, P., Jones, E. (2007) Warm-up and stretching in the prevention of muscular injury. Sports Medicine, 37, 1089-1099.

Yamakage, M., Iwasaki, S., Namiki, A. (2002) Evaluation of a newly developed monitor of deep body temperature. Journal of Anesthesia, 16, 354-357. 


\section{Figure Legends}

Figure 1: Changes (mean $\pm \mathrm{SD}$ ) in normalized iEMG (a) and torque (b) over 12 eccentric contractions for the warm-up (WU) and control (CON) conditions.

Figure 2: Changes (mean \pm SD) in muscle soreness (VAS scale) before (Pre), immediately after (Post), and 1-5 days following eccentric exercise for the warm-up (WU) and control $(\mathrm{CON})$ conditions.

Figure 3: Changes (mean $\pm \mathrm{SD}$ ) in maximal voluntary isometric contractions (MVC) torque before (Pre), immediately after (Post), and 1-5 days following eccentric exercise for the warm-up (WU) and control (CON) conditions. 


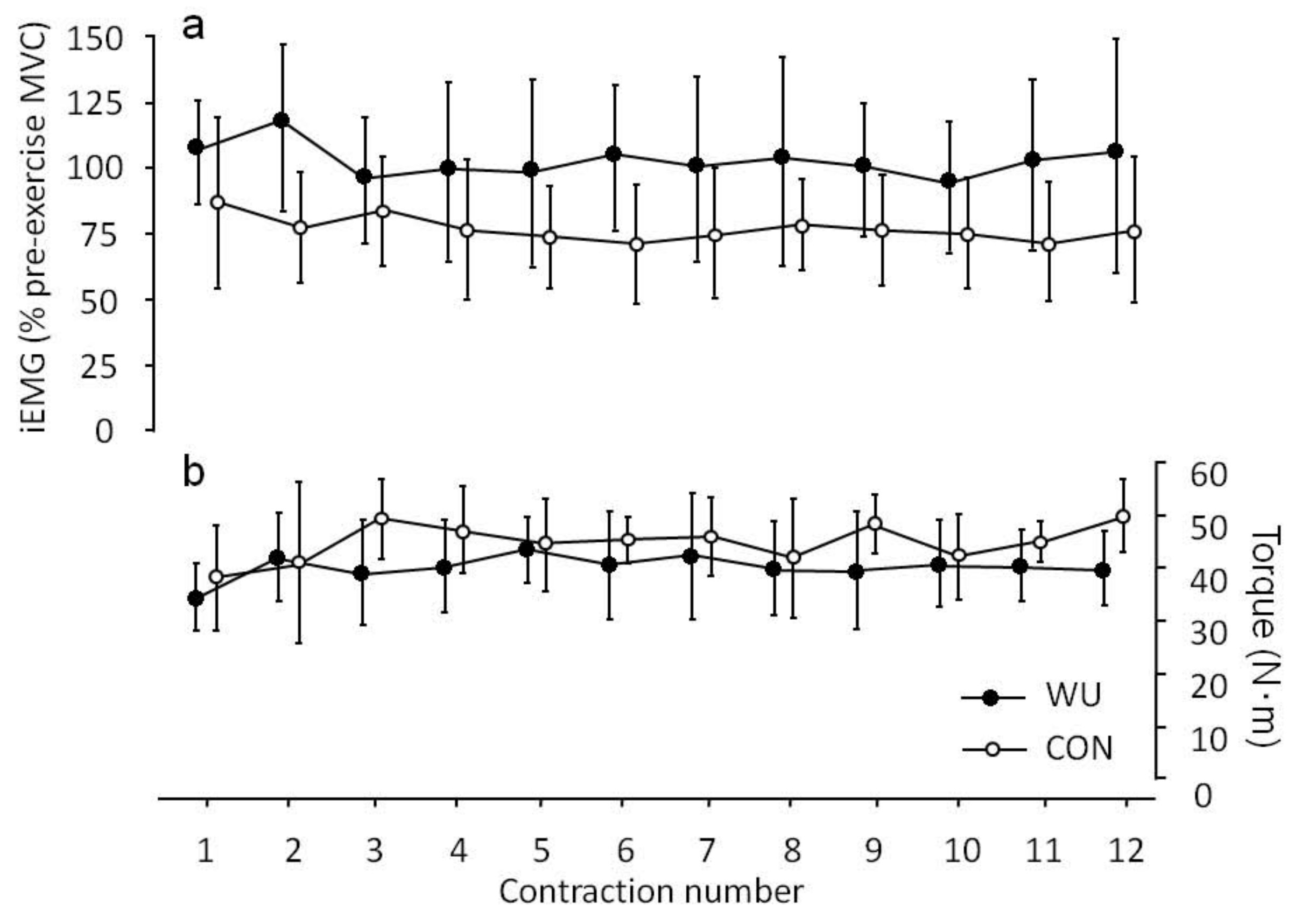




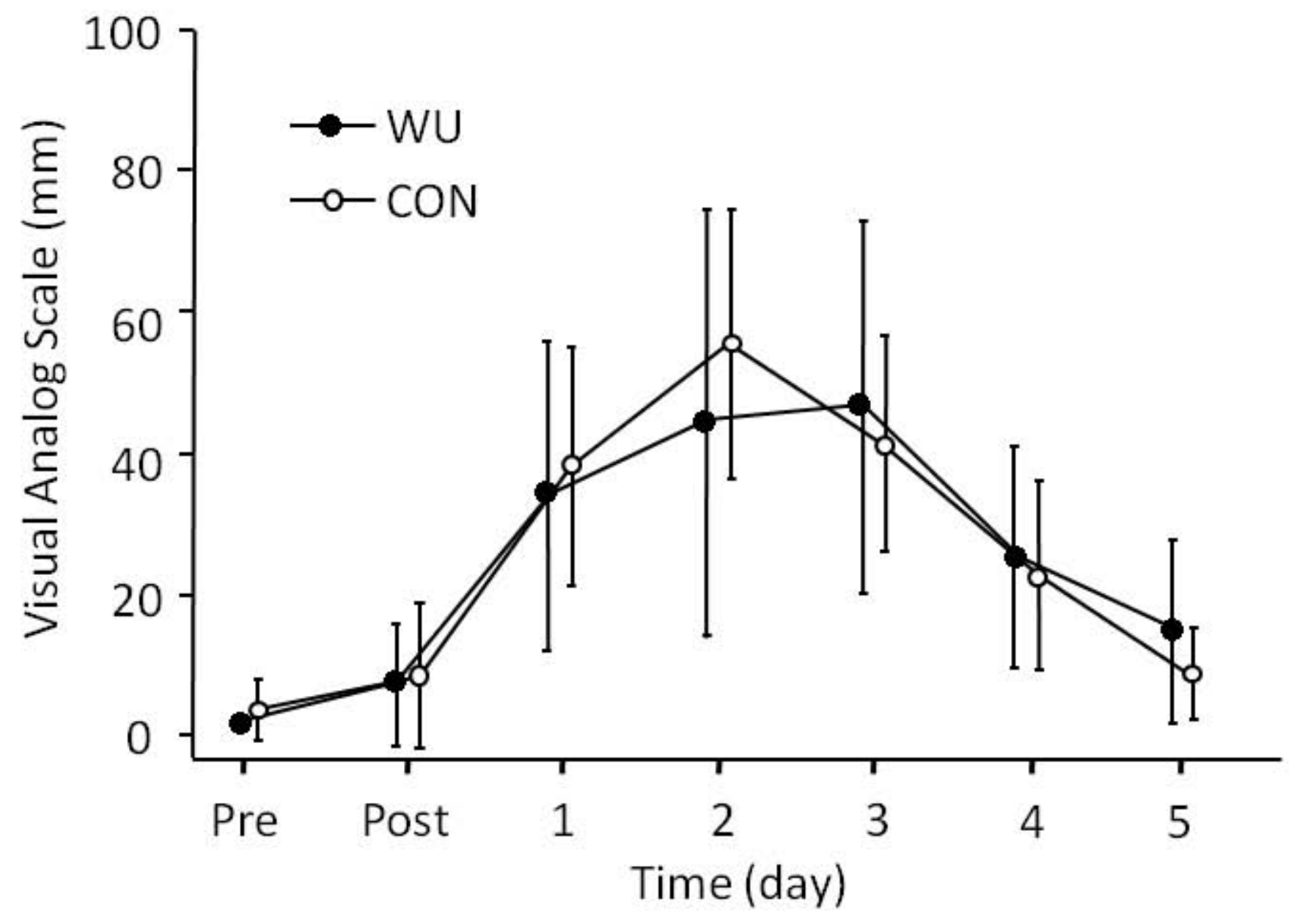




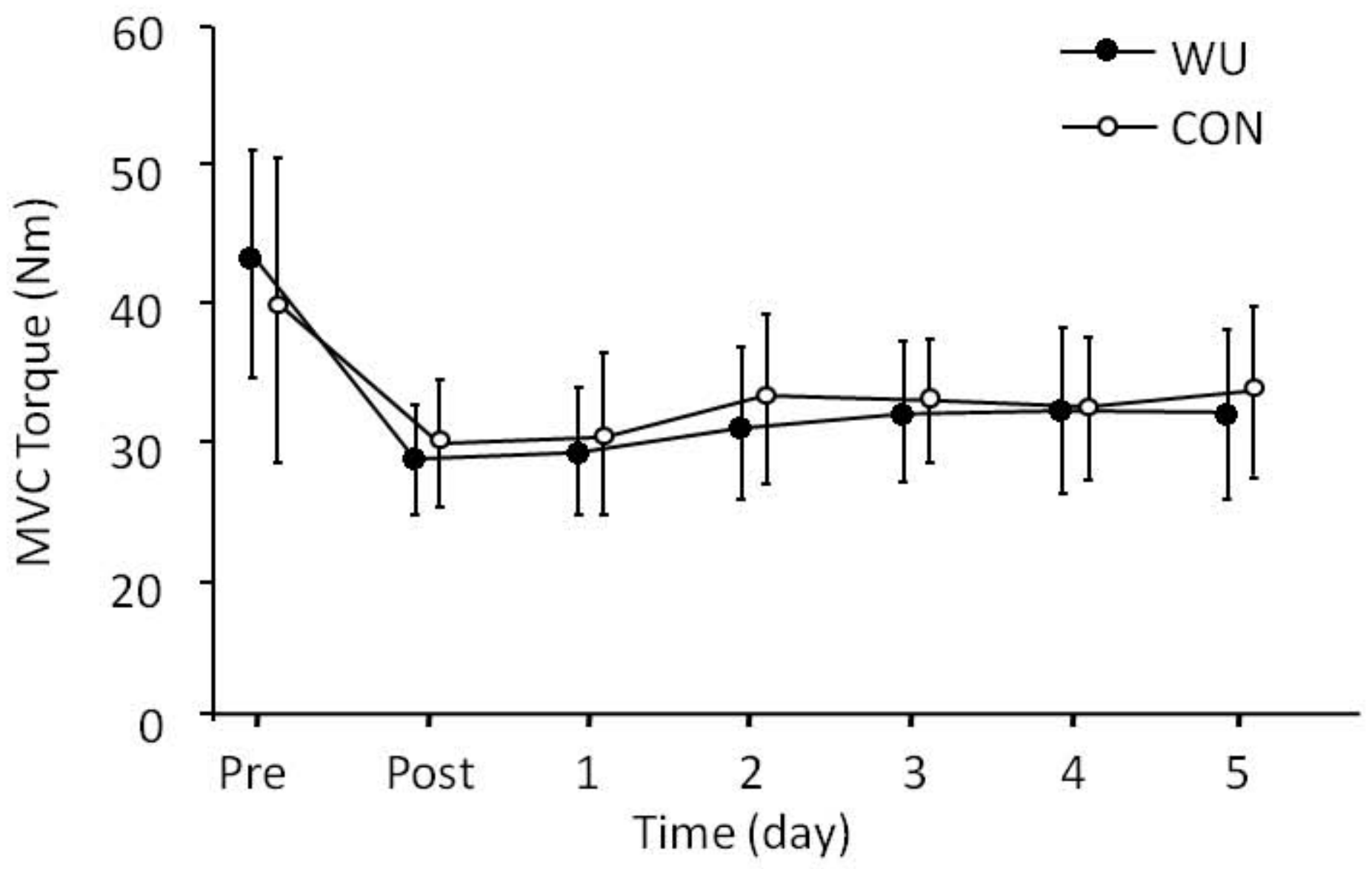

